\title{
Diarrhö bei Reiserückkehrern sorgfältig abklären!
}

Eine Reisediarrhö kann nicht nur den Urlaub verderben, sondern langfristige Folgen haben: Ein postinfektiöser Reizdarm kommt bei etwa fünf Prozent der Erkrankten vor.

Die Inzidenz von Reisediarrhö ist je nach Reiseland ja sehr variabel und reicht von 20 bis über $50 \%$ - „wobei für viele tropische Destinationen in den vergangenen Jahren ein Rückgang der Diarrhö-Häufigkeit beschrieben wurde", berichtete Professor Thomas Weinke vom Klinikum Ernst von Bergmann in Potsdam. Die entscheidende Therapiemaßnahme sei dann die orale Rehydratation, um Komplikationen zu verhindern.

„Antibiotika können den Krankheitsverlauf abkürzen, da es sich aber meist um eine Selbsttherapie im Reiseland handelt, muss der Reisende bei der reisemedizinischen Beratung zuvor sorgfältig über Indikation, Medikamente und den Einnahmemodus aufgeklärt werden", erinnerte Weinke beim 22. Forum Reisen und Gesundheit. Für eine solche Therapie böten sich Rifamycin oder Rifaximin (nicht resorbierbar) bei nicht-invasiven Erregern an, Azithromycin bei invasiven bakteriellen Erregern. Chinolone seien nicht mehr zu vertreten.

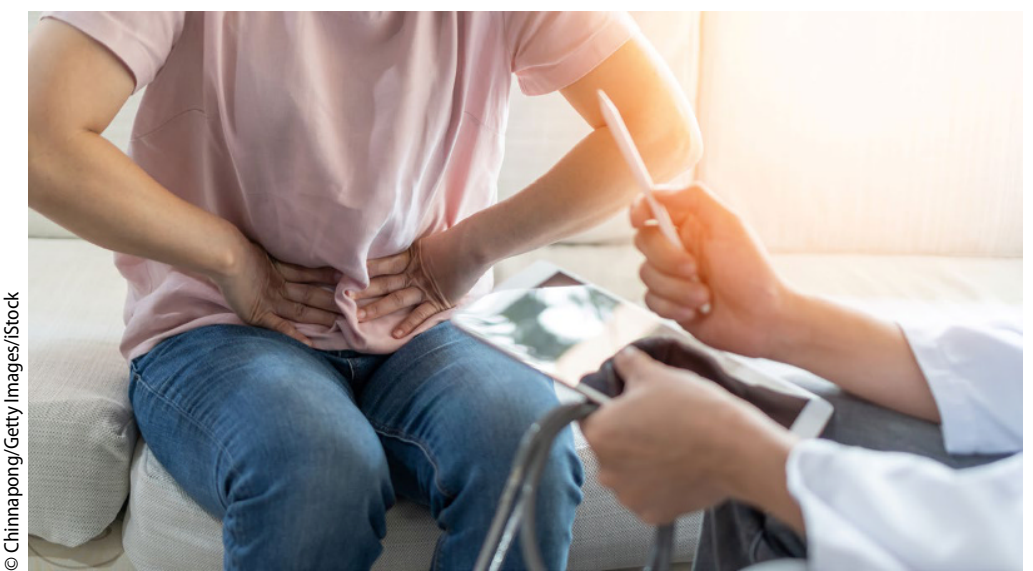

zeitung.de, veröffentlicht am 02.06.2021

\section{Untersuchung auf Parasiten}

„Auch bei Reiserückkehrern sind gastrointestinale Symptome das häufigste Problem, genauer gesagt, bei $45 \%{ }^{\prime \prime}$, führte Weinke aus. Bei nach Reiseende bestehender Diarrhö müsse eine genaue mikrobiologische Klärung durchgeführt werden - „dazu gehört auch die Untersuchung auf Parasiten, am wichtigsten sind dabei Amöben und Lamblien."

》) $45 \%$ der Reiserückkehrer berichten über gastrointestinale Symptome, am häufigsten über Diarrhö

Letztere sollten vor allem bei einer Krankheitsdauer von über 14 Tagen abgeklärt werden, bei Blutabgängen und/oder Fieber gelte es, eine Infektion mit Shigellen, Campylobacter und Salmonellen zu prüfen. Bei fieberhafter Diarrhö nach Tropenreise muss zudem im Rahmen einer Sofortdiagnostik eine Malaria ausgeschlossen werden.
$\Delta$ Der postinfektiöse Reizdarm kann auch noch mit deutlichem zeitlichem Abstand zur Reise auftreten
Der postinfektiöse Reizdarm gilt als Problem, das auch noch mit deutlichem zeitlichen Abstand zur Reise auftreten kann. Eine solche Symptomatik komme bei etwa fünf Prozent der Erkrankten vor, sagte Weinke.

\section{Nahrungsmittelhygiene hat höchste Priorität}

Für die Prophylaxe der Reisediarrhö und damit auch des postinfektiösen Reizdarms - hat das Prinzip der Nahrungsmittelhygiene höchste Priorität. Relevant ist die Prophylaxe vor allem für CED-Patienten, Kinder sowie ältere Menschen, multimorbide Patienten und solche unter Säureblockade.

„Eine Impfung gegen Reisediarrhö wäre zwar wünschenswert, ist allerdings bei den vielen auslösenden Pathogenen nicht realistisch", meinte Weinke. Zwar könne mit dem CholeraImpfstoff eine gewisse Kreuzprotektion erreicht werden, dies reiche aber nicht für eine generelle Impfempfehlung.

„Eine Prophylaxe mit Antibiotika ist eindeutig kontraindiziert, und für den Einsatz von Probiotika, Heilerde oder Homöopathika gibt es nur eine kaum vorhandene Evidenz", fasste Weinke die weiteren Optionen zusammen.

\section{Bericht: Anne Bäurle}

Hinweis des Verlags. Der Verlag bleibt in Hinblick auf geografische Zuordnungen und Gebietsbezeichnungen in veröffentlichten Karten und Institutsadressen neutral.

Schweiz. Gastroenterol. $2021 \cdot$ 2:152 https://doi.org/10.1007/s43472021-00055-x

(c) Springer-Verlag GmbH Austria, ein Teil von Springer Nature 2021 